\title{
ESTIMATION OF SHIP REPAIR TIME USING THE GENETIC ALGORITHM
}

\author{
Remus Zagan ${ }^{1}$ \\ Iwona Paprocka *2 \\ Mihaela-Greti Manea $^{3}$ \& Emill Manea ${ }^{4}$ \\ ${ }^{1}$ Constanta Maritime University, Faculty of Navigation and Naval Transport, Romania \\ ${ }^{2}$ Silesian University of Technology, Faculty of Mechanical Engineering, Gliwice, Poland \\ 3 "Ovidius" University of Constanta, Romania \\ ${ }^{4}$ Constanta Shipyard-Romania, Greece Branch Office \\ *Corresponding author: iwona.paprocka@polsl.pl (I. Paprocka)
}

\begin{abstract}
Ship maintenance is regulated by both the state and the classification society. The scope of maintenance works depends on the age of the ship and includes a dock, intermediate and special inspection. The problem is to estimate the reliable time of the ship maintenance and the downtime at the shipyard. The purpose of this article is to develop a more accurate model to predict a ship's overall maintenance time. A multiple linear regression model is developed to describe the impact of historical data on hull repair, painting time, piping, age, structural and hull plate replacement for ship maintenance. In the literature, the least squares method is used to estimate unknown regression coefficients. The original value of the article is the use of a genetic algorithm to estimate coefficient values of the multiple linear regression model. Necessary analysis and simulations are performed on the data collected for oil and chemical or product tankers. As a result, a significant improvement in the adequacy of the presented model was identified.
\end{abstract}

Keywords: Mean time of dry-docking, average repair time, maintenance duration prediction; maintenance duration estimation; ship maintenance; genetic algorithm.

\section{INTRODUCTION}

The goal of the shipyard is to ensure the supply of products and services for the period specified in the contract, in accordance with quality requirements and international standards. Customer satisfaction is monitored to optimise yard operations, from maintenance processes to ship delivery. Shipowners strive to reduce the maintenance period when ships are on low income. On the other hand, the yard is trying to shorten the repair period for dry docks and quays, to maximise revenues by contracting more ships. Maintenance works depend on the requirements of classification societies and the possibility of their performance by the shipyard.

One of the goals is to pre-define the maintenance work in the technical specification in order to limit any additional orders that arise during the maintenance, thus keeping the costs of the shipyards and vessels within the initially estimated budgetary margins. Another goal is to estimate the duration of maintenance in the technical specification, which depends on previously defined works. Any additional orders entail additional costs for both parties and result in extending the maintenance period of the dock. The ship yard needs additional manpower to cover additional orders for the ongoing projects, thus increasing the risk of adversely affecting the development of running parallel or sequential projects. For the shipowner, additional costs and delayed ship delivery time may have a negative impact on the continuation of the assumed commercial obligations.

The purpose of this paper is to develop a more accurate model to predict a ship's overall maintenance time. A multiple linear regression model is used to describe the effect of hull repair data, ship age, painting time, piping, structural and hull plate replacement on the time required for ship maintenance. The data on maintenance work was collected at Constanta Shipyard in Romania. Another objective is to identify the variables that have a significant impact on the ship's maintenance time and their inter-relationships. 


\section{LITERATURE REVIEW}

Shipowners carry out preventive maintenance to extend the life cycle of ships' machinery, power, equipment and vessels. A lot of effort is put into modelling the technical condition of the machines, machine elements and machine working conditions. Observation of the dependence of the operating parameters of machine elements and environmental parameters allows the prediction of the life cycle of the machine and planning the technical inspection of the machine at the right time. For example, the paper by [1] presented the possibility of determining the technical condition of lip seals on the basis that the proposed coefficient of correctness of the operation has been described. The authors proved the hypothesis that the quality of the oil affects the durability of lip seals and elaborated a maintenance system that is understandable to the crew, which is essential for increasing the durability of the ship. Familiarisation with adequate procedures can significantly contribute to the successful prevention of accidents [2]. When analysing the literature, two different opinions were identified.

On the one hand, to ensure the safe operation of a ship, the optimal interval of dry-docking inspections (Mean Time To Dry-docking) is estimated. Gong et al. [3] developed a conditionbased, decision-making approach for the inspection time. The probability distributions of the life-cycle costs include two components. The first component is the cost of ship maintenance, distinguishing dry-docking and structural renewal and, the second, is the cost of failure consequence. It is important to establish a cost-effective inspection time that allows the ship to stay longer in water, with an acceptable level of safety. The ship owner must decide in which sequence and when to carry out maintenance works of the ships. The article [4] presents a model of real state transitions of the ship main engine with states: (1) state of full serviceability, (2) state of partial serviceability, and (3) state of unserviceability. Empirical data concerning the ship's main engine are used for calculating limiting probabilities for the process. Thus, it is possible to choose between the following two decisions: (1) perform a relevant preventive service of the engine to restore its state and then perform the commissioned task within the time limit determined by the customer or, (2) perform the preventive service and start performing the commissioned task. Wu et al. [5] developed a component maintenance priority measure, which is used to select a component for maintenance. This metric can be adapted to decide which ship to select next from among simultaneous shipyard access requests, while one ship is undergoing maintenance. In general, the date of commencement of maintenance works on the ship depends on the condition of the ship [3] and the required frequency and duration of maintenance.

On the other hand, the duration of the ship repair (Mean Time of Dry-docking, Average Repair Time) is estimated, because it is in the interest of both shipowners and shipyards to make it as short as possible. Ship maintenance adversely affects the availability of operations of the ship and the shipyard. A ship repair yard must be aware, well in advance, of the total workload and resources needed to complete each job. Usually, managers use a guide book with the given man-hour totals for each trade and the work rate of each trade, when determining the timescale and daily loadings for carrying out the repairs [6]. The graphs are compiled from historical data by shipyard workload planners and used, to assist the forward planning of the yard. There is no standard model used by shipyard owners in the range of ship maintenance duration estimation. Naffisah et al. [7] searched for any patterns in different types of maintenance works in order to estimate the repair time. They proposed the Artificial Neural Network forecasting with backpropagation method, to upgrade the accuracy of maintenance duration predictions. Unfortunately, they obtained poorly accurate predictions due to the lack of a similar data pattern in the training data. The model is unable to make accurate predictions because there is a dissimilar or unrepresented data pattern in the training data. The high level of variance is explained by the influence of external factors, such as weather conditions and materials or maintenance employee availability. Surjandari and Novita [8] used the metod of Data Mining, Classification and Regression Trees to estimate the duration time of a ship's dry-docking for maintenance. They were searching for a hidden pattern that shows the relationship between maintenance work and maintenance duration and classified three dry-docking works which influence maintenance duration: propeller, washing and plate. They achieved four classes of maintenance duration, with a different liner model for each class. For example, for the propeller, the linear model to estimate time is classified based on the washing task. If the washing surface is less than a given threshold, the maintenance duration is estimated based on the plate task and so on. Unfortunately, the presented model achieved high error values. The least squares method for estimating unknown regression coefficients and describing the maintenance data with a low coefficient of determination was proposed [9]. Also, Dev and Sasha [10] used the method of least squares to estimate the regression coefficients for the model of a multiple linear regression, describing the function of ship data with respect to age, deadweight, hull coating, piping, structural steel, and tank coating, to estimate a ship repair duration time. The authors concluded that the adequacy of the model was low. In other words, a significant amount of variation in the response of the dependent variable (maintenance duration) is due to high differences in the independent variables. Terełko [11] presented a control model for the maintenance level of the main marine engines, with the use of regression analysis. Factors influencing the level of maintenance were: component weight, number of joints, component temperature, staff attitude. Regression functions were also achieved for the fuel system, cooling system, lubrication system, supercharging system, starting system, piston and crankshaft system, and cylinder and frame units, with very high coefficients of multiple determination (0.57-0.97). Bouayed et al. [12] proposed a parametric regression model to estimate maintenance costs based on historical data from Royal Canadian Navy ships. The cost of maintenance is the natural logarithm of the ship's annual maintenance cost, the class of the ship and the sea-going days recorded for the ship in the current year. The cost of maintenance depends 
on physical factors (weight, length), performance factors (maximum speed), demand (frequency of use of the system), and qualitative factors (system condition).

The literature review showed that only a few articles dealt with the problem of estimating the average ship repair time by observing the relationship between the data on the duration of dry dock works and the actual duration of the repair. This paper presents a new approach to estimating the average ship repair time for managers, to assist in advanced planning in a shipyard.

\section{GOALS AND APPROACHES}

The original value of this paper is the application of a genetic algorithm (GA) for estimation of the values of regression coefficients in the multiple linear regression model. The aim is to develop a method of estimating the average ship repair time that achieves the trend function with good compliance with historical data, i.e. the function with the highest value of multiple determination coefficients and the lowest value of standard deviation.

For the estimation of the average ship repair time problem, GA is proposed. GA is a popular approach for solving different optimisation problems $[13,14,15]$ and is a meta-heuristic method that gives a satisfying solution, but not an optimal one, in a reasonable amount of time. In the conditions of a competitive market, managers do not need the optimal solution, they need it to be good enough that fulfil the given constraints and criteria. In the paper by [16], GA is compared with the Multi-Objective Immune Algorithm (MOIA) and Clonal Selection Algorithm (CSA) when investigating the influence of basic schedules on the obtainment of stable and robust schedules, in the case of disturbance occurrence. The GA turns out to be a better method for generating predictive schedules.

This paper continues the problem of searching for a good enough solution for the problem of the average ship repair time estimation using GA. The proposed genetic algorithm uses mutation and selection mechanisms to explore and exploit a solution space; in other words, to search a set of regression coefficients. The regression coefficients describe relationships between data on maintenance duration time and data on maintenance works. The chromosome encodes a solution that is a set of values for the regression coefficients. The weighted function of the standard deviation and the multiple coefficient of determination assess the affinity of the chromosome for environmental conditions. Necessary analysis and simulations are performed on the data collected for oil and chemical or product tankers.

The paper is organised as follows: the next section presents a model for estimating the duration of a repair. Regression coefficients estimation, using the Solver appendix in Microsoft Office Excel, is presented in Section 3. The genetic algorithm for estimation of the regression coefficients is described in Section 4. Section 5 contains the necessary analyses and experimental test results related to the research on the application of the GA. The paper concludes with a brief summary of the results (Section 6).

\section{THE MODEL OF REPAIR TIME ESTIMATION}

The problem of estimating the duration of the maintenance takes into account the following planned works: hull repair $(\mathrm{Hr})$, painting $(\mathrm{Pa})$, structure renovation $(\mathrm{Sr})$, panel renovation $(P l)$, pipe repair $(P r)$ in resources: dock (docking duration) and quayside (quaytime). In addition to the type of repair tasks, the age of the ship (As) also affects maintenance duration $(R T)$ (both docking and quayside times). Data were collected from the Constanta Shipyard in Romania.

A shipyard usually carries out simultaneous construction and/or repair works for several ongoing projects. Therefore, it optimized human and technical resources in the scope of implemented processes and loading activities. When deciding to apply for a project, a shipyard knows its production capacity very well, which consists of human resources and technical equipment, which is approximately constant as a numerical value. In emergency situations, only the yard mobilses workers and equipment from one project to another, as doing so would disrupt the yard's flow of activities and generate unacceptable delays for the beneficiaries.

The shipyard is interested in estimating the docking time of ships for repair works. in order to plan the loading of the pine/floating docks. The authors of this study assume that this estimate depends on the content of the technical specification of the works received from the beneficiary/owner of the ship, and not on the human and technical capabilities of the shipyard. The construction of the database from the Constana Shipyard's portfolio of works, and the simulation presented in this paper, was carried out on the basis of the hypothesis that the yard has the optimal (considered to be a constant) number of employees and equipment for repair works. The variable that the shipyard takes into account, in the analyses carried out in order to assess the possibility of repair works, is the content of the technical specification for Repair Works received by the shipyard (expressed in square metres, cubic metres, running metres, tonnes, etc.).

To summarise, knowing its human resources and technical equipment, and considering them to be constant for a given project, the yard is interested in estimating the time of docking and stopping the ship for repair works, depending on the content of the technical specification for Repair Works (this content is a variable of interest to the shipyard). The authors try to answer this question in the body of the paper.

In order to establish the relationship between modes and repair time, historical data is collected both for dependent (repair duration) and independent variables (ship repair works, age). Multiple linear regression analysis is adopted from [10], to identify the mathematical relationship between modes and repair duration.

The multiple linear regression model is based on the equation:

$$
\begin{gathered}
b_{0}+b_{1} \cdot H r_{i}+b_{2} \cdot P a_{i}+b_{3} \cdot S r_{i}+b_{4} \cdot P l_{i}+ \\
b_{5} \cdot P r_{i}+b_{6} \cdot A s_{i}=R T_{i}
\end{gathered}
$$


where:

$b_{0}, b_{1}, \ldots, b_{k}$ - regression coefficients, $j \in[1,2, \ldots, k]$,

$k$ - size of independent variables, $k=7$,

$H r_{i}, P a_{i}, S r_{i}, P l_{i}, P r_{i}, A s_{i}, R T_{i}$ - variables, $i \in[1,2, \ldots, n]$,

$n$ - sample size, $n=108$ for all types of ships, $n=9$ for each

type separately,

$\mathrm{Hr}$ - hull repairing [m2],

$P a_{i}$ - painting $[\mathrm{m} 2]$,

$\mathrm{Sr}_{i}$ - structure renovation $[\mathrm{kg}]$,

$\mathrm{Pl}_{i}$ - plates renovation $[\mathrm{kg}]$,

$\mathrm{Pr}_{i}$ - pipes repairing $[\mathrm{m}]$,

$A s_{i}$ - age of the ship [years],

$R T_{i}$ - maintenance time [days].

By applying the least squares method [10] the formulas are achieved:

$$
\begin{aligned}
& n \cdot b_{0}+b_{1} \sum_{i=1}^{n} H r_{i}+b_{2} \sum_{i=1}^{n} P a_{i}+b_{3} \sum_{i=1}^{n} S r_{i}+b \sum_{i=1}^{n} P l_{i}+ \\
& b_{5} \sum_{i=1}^{n} \operatorname{Pr}_{i}+b \sum_{i=1}^{n} A s_{i}=\sum_{i=1}^{n} R T_{i} \\
& b_{0} \sum_{i=1}^{n} H r_{i}+b_{1} \sum_{i=1}^{n} H r_{i}^{2}+b_{2} \sum_{i=1}^{n} H r_{i} \cdot P a_{i}+ \\
& b_{3} \sum_{i=1}^{n} H r_{i} \cdot S r_{i}+b \sum_{i=1}^{n} H r_{i} \cdot P l_{i}+b \sum_{i=1}^{n} H r_{i} \cdot \operatorname{Pr}_{i}+ \\
& b_{6} \sum_{i=1}^{n} H r_{i} \cdot A s_{i}=\sum_{i=1}^{n} H r_{i} \cdot R T_{i} \\
& b_{0} \sum_{i=1}^{n} P a_{i}+b_{1} \sum_{i=1}^{n} P a_{i} \cdot H r_{i}+b \sum_{i=1}^{n} P a_{i}^{2}+ \\
& b_{3} \sum_{i=1}^{n} \mathrm{~Pa}_{i} \cdot \mathrm{Sr}_{i}+b_{4} \sum_{i=1}^{n} \mathrm{~Pa}_{i} \cdot \mathrm{Pl}_{i}+b_{5} \sum_{i=1}^{n} \mathrm{~Pa}_{i} \cdot \mathrm{Pr}_{i}+ \\
& b_{6} \sum_{i=1}^{n} P a_{i} \cdot A s_{i}=\sum_{i=1}^{n} P a_{i} \cdot R T_{i} \\
& b_{0} \sum_{i=1}^{n} S r_{i}+b_{1} \sum_{i=1}^{n} S r_{i} \cdot H r_{i}+b \sum_{i=1}^{n} S r_{i} \cdot P a_{i}+ \\
& b_{3} \sum_{i=1}^{n} S r_{i}^{2}+b_{4} \sum_{i=1}^{n} S r_{i} \cdot P l_{i}+b_{5} \sum_{i=1}^{n} S r_{i} \cdot \operatorname{Pr}_{i}+ \\
& b \sum_{i=1}^{n} S r_{i} \cdot A s_{i}=\sum_{i=1}^{n} S r_{i} \cdot R T_{i} \\
& b_{0} \sum_{i=1}^{n} P l_{i}+b \sum_{i=1}^{n} P l_{i} \cdot H r_{i}+b \sum_{i=1}^{n} P l_{i} \cdot P a_{i}+ \\
& b_{3} \sum_{i=1}^{n} P l_{i} \cdot S r_{i}+b \sum_{i=1}^{n} P l_{i}^{2}+b \sum_{i=1}^{n} P l_{i} \cdot P r_{i}+ \\
& b \sum_{i=1}^{n} P l_{i} \cdot A s_{i}=\sum_{i=1}^{n} P l_{i} \cdot R T_{i} \\
& b_{0} \sum_{i=1}^{n} \operatorname{Pr}_{i}+b_{1} \sum_{i=1}^{n} \operatorname{Pr}_{i} \cdot H r_{i}+b_{2} \sum_{i=1}^{n} \operatorname{Pr}_{i} \cdot P a_{i}+ \\
& b \sum_{i=1}^{n} \operatorname{Pr}_{i} \cdot S r_{i}+b \sum_{i=1}^{n} \operatorname{Pr}_{i} \cdot P l_{i}+b \sum_{i=1}^{n} \operatorname{Pr}_{i}^{2}+ \\
& b \sum_{i=1}^{n} \operatorname{Pr}_{i} \cdot A s_{i}=\sum_{i=1}^{n} \operatorname{Pr}_{i} \cdot R T_{i}
\end{aligned}
$$

$$
\begin{gathered}
b_{0} \sum_{i=1}^{n} A s_{i}+b_{1} \sum_{i=1}^{n} A s_{i} \cdot H r_{i}+b \sum_{i=1}^{n} A s_{i} \cdot P a_{i}+ \\
b_{3} \sum_{i=1}^{n} A s_{i} \cdot S r_{i}+b_{4} \sum_{i=1}^{n} A s_{i} \cdot P l_{i}+b_{5} \sum_{i=1}^{n} A s_{i} \cdot P r_{i}+ \\
b_{6} \sum_{i=1}^{n} A s_{i}^{2}=\sum_{i=1}^{n} A s_{i} \cdot R T_{i}
\end{gathered}
$$

The historical data on $\mathrm{Hr}_{i}, \mathrm{~Pa}_{i}, \mathrm{Sr}_{i}, \mathrm{Pl}_{i}, \mathrm{Pr}_{i}, A s_{i}, \mathrm{RT}_{i}$ is applied in the above equations, simultaneously. Regression coefficients $b_{0}+b_{1}, \ldots, b_{k}$ are estimated using the Genetic Algorithm presented in the next section. The presented model assumes the selection of variables that have the greatest impact on the average repair time. The adequacy of the presented model is calculated with:

1) standard deviation

$$
s=\left[\frac{1}{n-(\mathrm{k}+1)} \sum_{i=1}^{n}\left(y_{i}-\hat{y}_{i}\right)^{2}\right]^{0.5} \quad i \in[1, \ldots, n]
$$

2) coefficient of multiple determination

$$
R^{2}=\frac{\sum_{i=1}^{n}\left(\hat{y}_{i}-\bar{y}_{i}\right)^{2}}{\sum_{t=1}^{n}\left(y_{i}-\bar{y}_{i}\right)^{2}}, R^{2} \in[0,1]
$$

where:

$y_{i}-$ actual value of variable $\mathrm{Y}$ in period $i$,

$\hat{y}_{i}$ - theoretical value of the $Y$ variable resulting from the model in period $i$,

$\bar{y}_{i}$ - mean value of the variable $\mathrm{Y}$ in the time series of length $n$, $k$ - number of variables explaining the model,

$k+1-$ number of model parameters.

The multiple determination coefficient measures the compliance of the trend function with historical data on $H r_{i}, \mathrm{~Pa}_{i}, \mathrm{Sr}_{i}, \mathrm{Pl}, \mathrm{Pr}, \mathrm{As}, \mathrm{RT}_{i}$. To estimate the average docking duration, the function with the highest value of the multiple determination coefficient (10) and the lowest value of standard deviation (9) is assumed.

\section{REGRESSION COEFFICIENTS ESTIMATION USING THE SOLVER APPENDIX OF MICROSOFT OFFICE EXCEL}

The presented model was veriffied with the Solver Appendix in Microsoft Office Excel. The following hypotheses were tested: - the repair time is proportional to variables: $\mathrm{Hr}, \mathrm{Pa}, \mathrm{Sr}, \mathrm{Pl}, \mathrm{Pr}$, $A s_{i}$, taking into account data collected for all types of ships, - the repair time is proportional to variables: $\mathrm{Hr}_{i}, \mathrm{~Pa}_{i}, \mathrm{Sr}_{i}, \mathrm{Pl}_{i}$, $\operatorname{Pr}_{i}, A s_{i}$, taking into account data collected for Crude Oil Tankers and for Chemical/Products Tankers.

- the repair time is proportional to variables: Hri, Pai, taking into account data collected for Crude Oil Tankers.

Taking into account the historical observations of $\mathrm{Hr}_{i}, \mathrm{~Pa}_{i}$, $S r_{i}, P l_{i}, P r_{i}$, and $A s_{i}$, for $n=108$ ships, the Solver Appendix estimates values of regression coefficient $b_{0}+b_{1}, \ldots, b_{k}$. The achieved regression equation is: 


$$
\begin{gathered}
108 \cdot 15.26804-0.00026 \sum_{i=1}^{n} H r_{i}+0.000076557 \sum_{i=1}^{n} P a_{i}+ \\
0.000189 \sum_{i=1}^{n} S r_{i}-0.000036 \sum_{i=1}^{n} P l_{i}-0.00303 \sum_{i=1}^{n} \operatorname{Pr}_{i}+ \\
0.000178 \sum_{i=1}^{n} A s_{i}=\sum_{i=1}^{n} R T_{i}
\end{gathered}
$$

Although the multiple determination coefficient $R^{2}$ equals 0.42 and the standard deviation is low $(s=2.78)$, the resulting equation cannot be accepted. The high value of parameter $b_{0}(15.26804)$ means that the value of repair time is low, related with variables: $H r_{i}, P a_{i}, S r_{i}, P l_{i}, \mathrm{Pr}_{i}$, and $A s_{i}$. The average ship repair time depends on the pipeline repair $\left(P r_{i}\right)$ to a large extent, since the value of $b_{5}$ is the highest $(0.00303)$ compared to the values of $b_{1}, b_{1}, b_{1}, b_{2}, b_{3}, b_{4}$, and $b_{6}$ (11).

Taking into account the historical observations of $\mathrm{Hr}_{i}$, $P a_{i}, S r_{i}, P l_{i}, P r_{i}$, and $A s_{i}$ for the Crude Oil Tanker, the Solver Appendix estimates values of regression coefficient $b_{0}, b_{1}, \ldots, b_{6}$. The achieved regression equation is:

$$
\begin{gathered}
9 \cdot 14.99894-0.00123 \sum_{i=1}^{n} \mathrm{Hr}_{i}+0.00096496 \sum_{i=1}^{n} \mathrm{~Pa}_{i}- \\
0.0000578 \sum_{i=1}^{n} S r_{i}-0.000071 \sum_{i=1}^{n} P l_{i}-0.000386 \sum_{i=1}^{n} \mathrm{Pr}_{i}+ \\
0.046164 \sum_{i=1}^{n} A s_{i}=\sum_{i=1}^{n} R T_{i}
\end{gathered}
$$

The achieved equation is more adequate for Crude Oil Tankers than for all ships. The coefficient of multiple determination is good, $R^{2}=0.79$, but the standard deviation is high, $s=9.97$. The average repair time equals 15.33 days but the time may vary by 10 days. The high value of parameter means that the repair duration is low, related with variables: $H r_{i}, P a_{i}, S r_{i}, P l_{i}, P_{i}$, and $A s_{i}$. The average repair time depends on the age of the ship (As), to the greatest extent.

Taking into account the historical observations of $\mathrm{Hr}_{i}, \mathrm{~Pa}_{i}$, $\mathrm{Sr}_{i}, \mathrm{Pl}_{i}, \mathrm{Pr}_{i}$, and $A s_{i}$ for the one type of Chemical/Products Tanker, the Solver Appendix estimates values of regression coefficient $b_{0}, b_{1}, \ldots, b_{6}$. The achieved regression equation is:

$$
\begin{gathered}
9 \cdot 19.42467-0.00048 \sum_{i=1}^{n} H r_{i}+0.000053258 \sum_{i=1}^{n} P a_{i}+ \\
0.0000903 \sum_{i=1}^{n} S r_{i}+0.0063044 \sum_{i=1}^{n} P l_{i}-0.00006 \sum_{i=1}^{n} \mathrm{Pr}_{i}- \\
0.15224 \sum_{i=1}^{n} A s_{i}=\sum_{i=1}^{n} R T_{i}
\end{gathered}
$$

The achieved equation is more adequate for Chemical/ Products Tankers. The coefficient of multiple determinations is $R^{2}=0.67$ and the standard deviation is 3.98. The average repair time is 19.55 days, but can vary by 4 days. The average repair time depends on the age of the ship (As) to the greatest extent.

Considering only the historical observations regarding $\mathrm{Hr}_{i}$ and $\mathrm{Pa}_{i}$, for 9 Crude Oil Tankers, the Solver Appendix estimates values of regression coefficient $b_{0}, b_{1}, b_{2}$. The achieved regression equation is:

$$
\begin{gathered}
9 \cdot 0.000701+0.000541 \sum_{i=1}^{n} H r_{i}+ \\
0.0000353 \sum_{i=1}^{n} P a_{i}=\sum_{i=1}^{n} R T_{i}
\end{gathered}
$$

The coefficient of multiple determination is very high, $R^{2}=0.99$, and the standard deviation is very low, $s=0.63$, thus the achieved equation fits the historical data. The average ship repair time depends on the hull repair time $(\mathrm{Hr})$ to the greatest extent.

\section{REGRESSION COEFFICIENT ESTIMATION USING THE GENETIC ALGORITHM}

The regression coefficient values are also searched using the Genetic Algorithm, for the problem of estimating the average repair time for all types of ships. The following hypothesis is tested:

- the repair time is proportional to variables: $\mathrm{Hr}_{i}, \mathrm{~Pa}_{i}, \mathrm{Sr}_{i}$,

$P l_{i}, P r_{i}$, and $A s_{i}$, taking into account data collected for all types of ships.

The GA consists of the following modules: data interface, individual encoding, genetic optimisation and selection, and individual decoding.

In the GA, an individual represents a vector of regression coefficient values for the problem of estimation of the mean repair time of a ship, while a fitness function is a measure used to assess the adequacy of the presented model in relation to historical data. The fitness function of an individual (15) is the weighted function of the coefficient of multiple determination (9) and standard deviations (10):

$$
F\left(\rho_{\eta}\right)=0.5 \cdot\left[1-\frac{R\left(\rho_{\eta}\right)^{2}}{R\left(\rho_{\eta}^{*}\right)^{2}}\right]+0.5 \cdot \frac{s\left(\rho_{\eta}\right)}{s\left(\rho_{\eta}^{*}\right)}
$$

where:

$s\left(\rho_{\eta}^{*}\right)$ - the maximum standard deviation achieved by chromosome $\rho^{*}{ }_{\eta}$ in iteration

$R\left(\rho^{*}{ }_{\eta}\right)^{2}$ - the maximum coefficient of multiple determination achieved by chromosome $\rho^{*}$.

The objective is to achieve the vector of regression coefficients with minimal value (15).

The pseudo code of the GA is presented in Fig. 1. The steps of the algorithm are explained in the following subsections.

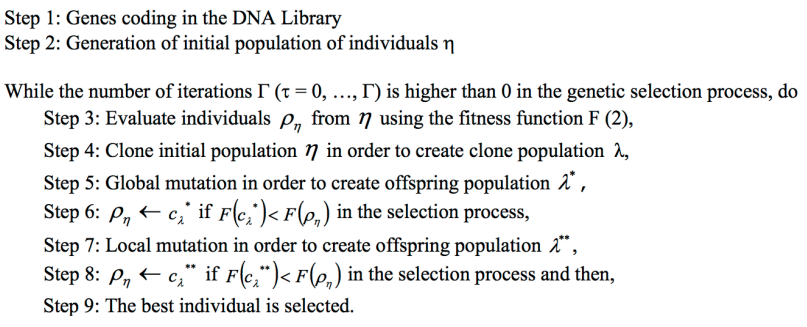

Fig. 1. Pseudo code of the GA algorithm

\section{ENCODING AND DECODING}

The complexity of the problem of estimating the average repair time for ships requires sophisticated coding practice for an individual, including regression coefficient values and a positive or negative sign before a coefficient. Each individual $\rho_{\eta}$ 
is represented by seven sub-chromosomes, one sub-chromosome for each regression coefficient. Each sub-chromosome is created by six genes, randomly selected from the DNA Library (Table 1). Each individual chromosome represents a binary gene for a sign, a floating point gene for the number of zeros after the decimal point, and an integer gene for the coefficient before and after the decimal point. By scanning the chromosome code from left to right, estimated values of regression coefficients $b_{0}, b_{1}, \ldots, b_{6}$ are entered into $\mathrm{Eq}(11)$.

Tab. 1. The DNA Library for each sub-chromosome

\begin{tabular}{|c|c|}
\hline$V=[-1,1]$ & $\leftarrow$ random generation of a gene for a sign \\
\hline$D=[0,1]$ & $\begin{aligned} \leftarrow \text { random gene generation for yes or no } \\
\text { decisions for integer values }\end{aligned}$ \\
\hline$J=[1, \ldots, 20]$ & $\begin{aligned} \leftarrow \text { random generation of a gene for } \\
\\
\text { a coefficient value before the decimal point }\end{aligned}$ \\
\hline$C=[0,1]$ & $\begin{aligned} \leftarrow \text { random generation of a gene for yes or no } \\
\text { decisions for fractional values }\end{aligned}$ \\
\hline $\begin{array}{l}E=[0.1,0.01,0.001, \\
0.0001,0.00001, \\
0.000001,0.0000001 \\
E=[[0.1,0.01,0.001, \\
0.0001,0.00001, \\
0.000001,0.0000001]]\end{array}$ & $\begin{aligned} \leftarrow \text { random generation of a gene for a number } \\
\text { of zeros after the decimal point }\end{aligned}$ \\
\hline$F=[1, \ldots, 999]$ & $\begin{aligned} \leftarrow \text { random generation of a gene for } \\
\text { a coefficient value after zeros }\end{aligned}$ \\
\hline
\end{tabular}

In order to transform an individual into a workable multiple linear regression equation, each sub-chromosome is decoded into a regression coefficient. Consider the first row of the matrix $\rho_{j, \eta}$ which codes regression coefficient $b_{0}$ (Table 2). Starting from the left to right, the first gene codes sign “+”, second gene codes a multiplier for the value before the decimal point, the third gene codes the value of the coefficient before the decimal point. The following gene $(\mathrm{C})$ codes a multiplier for the value after the decimal point, gene E codes a number of zeros after the decimal point, and the last gene codes the value after zeros for the coefficient.

Decoding each sub-chromosome involves solving the following procedure:

$$
\rho_{j, \eta}=(V) \cdot(D \cdot J+(C \cdot(E \cdot F)))
$$

Consider the first sub-chromosome, which codes value for $b_{0}$,

$$
\begin{gathered}
\rho_{j, \eta}=(V) \cdot(D \cdot J+(C \cdot(E \cdot F)))= \\
+1 \cdot(1 \cdot 15+(1 \cdot(0.001 \cdot 289)))
\end{gathered}
$$

value 15.289 is substituted instead of $b_{0}$ into Eq. (2).
Tab. 2. An individual (chromosome) randomly generated from the DNA Library

\begin{tabular}{|c|c|c|c|c|c|c|}
\hline$\rho_{j, \eta}$ & $V$ & $D$ & $J$ & $C$ & $E$ & $F$ \\
\hline$b_{0}$ & 1 & 1 & 15 & 1 & 0.001 & 268 \\
\hline$b_{1}$ & 0 & 0 & 11 & 1 & 0.00001 & 26 \\
\hline$b_{2}$ & 1 & 0 & 7 & 1 & 0.0000001 & 765 \\
\hline$b_{3}$ & 1 & 0 & 9 & 1 & 0.00001 & 189 \\
\hline$b_{4}$ & 0 & 1 & 3 & 1 & 0.000001 & 36 \\
\hline$b_{5}$ & 0 & 0 & 2 & & 0.00001 & 303 \\
\hline$b_{6}$ & 1 & 0 & 1 & 1 & 0.000001 & 178 \\
\hline
\end{tabular}

After decoding all sub-chromosomes, values for the parameters $b_{1}, b_{1}, b_{1}, b_{2}, b_{3}, b_{4}, b_{5}$ and $b_{6}$ in Eq. (11) are achieved.

\section{INITIALISATION}

Genes, stored in the DNA Library, represent signs (+ or -), yes or no decisions for a multiplier, a number of zero positions and values in the problem of the ship maintenance time estimation. An individual's sub-chromosome consists of the $V, D, J, C, E$, and $F$ genes. A set of randomly generated regression coefficients (sub-chromosomes) represents an individual (chromosome) in the initial population.

\section{CHROMOSOME DIFFERENTIATION AND SELECTION}

The parent pool is created by copying the initial population. The two point mutation procedure is proposed in order to construct new regression coefficients in the chromosome differentiation process. The intensity of the effect of the mutation procedure is controlled by defining a series of subchromosomes and genes that are subject to the mutation procedure.

The two-point mutation procedure begins by selecting two subchromosomes from $V, D, J, C, E$, and $F$ and one gene for each subchromosome. Selected genes are removed from the parent's chromosome. Offspring are produced by copying the rest of the genes into their chromosome positions. As a result, the genes required to complete offspring are selected randomly from the DNA library.

Let us assume that sub-chromosomes $b_{0}$ and $b_{1}$ undergo the two-point mutation procedure. Two genes were selected: $D$ and $F$. The selected genes were removed from the chromosome of the parent. New genes $D$ and $F$ were copied from the DNA Library in the corresponding positions of the offspring's chromosome, as presented in Fig. 2.

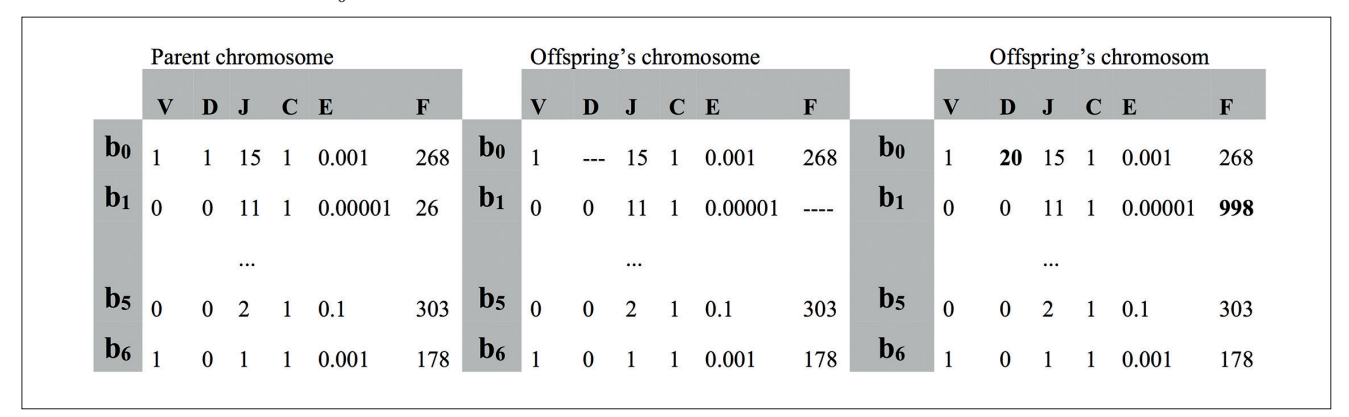

Fig. 2. The chromosome of the offspring generation with random selection of two genes, $D$ and $F$ 
The decoding procedure is then applied and the multiple regression equation for each person is calculated. In the elite selection, the best individual does not change from a pair: parent and offspring.

After the selection, the parent undergoes the procedure of single point mutation. In a single point mutation, the $F$ gene from a randomly selected sub-chromosome is replaced with a random gene from a DNA library. Due to the use of the single point mutation, the emphasis on genetic material loss is low. The elite choice is also repeated. The best individuals remain unchanged and will survive to the next generation.

\section{TERMINAL CONDITION}

The execution of multiple iterations is a termination condition. The best solution (optimal or close to optimal) is in the last generation.

\section{COMPUTER SIMULATION}

In this section, the problem of estimating ship maintenance time is examined using a genetic algorithm written in Borland $\mathrm{C}++$. Historical data on both dependent (repair time) and independent variables (type, age of a ship) to establish the relationship between modes and repair time was used. A multiple linear regression analysis is applied in order to identify the mathematical relationship between modes and repair time (1-8). The relationship between both dependent and independent variables is described by the vector of regression coefficients. The fitness function measures the adequacy of the presented model to historical data.
Computer experiments are run for alternative values of key parameters, such as the size of the initial population (chn), number of iterations (in) and number of mutation points ( $\mathrm{mn}$ ). The values of the parameters are varied including the initial population $\{100,150\}$, number of iterations $\{50\}$ and number of mutation points $\{1,2\}$. Only a small number of instances are examined from the set of parameter values to evaluate the GA, with the objective of increasing the coefficient of multiple determinations. A small number of experiments indicate the need to change the coding procedures presented in this article.

The general function of the GA is maintained, that is, the average quality of population improves from generation to generation, as shown in Fig. 3, especially for experiments $\{\mathrm{chn}=100, \mathrm{mn}=1, \mathrm{in}=50\}$ and $\{\mathrm{chn}=100, \mathrm{mn}=2$, in $=50\}$.

Two computer simulations (cs $=2$ ) are performed for the input data sets: $\{\mathrm{chn}=100, \mathrm{mn}=1, \mathrm{in}=50\}$ and $\{\mathrm{chn}=100$, $m n=2$, and $n=50\}$. Comparing the mean fitness function obtained for the simulations (Fig. 3, red and green lines for the first data set, grey and blue lines for the second data set), the following conclusion can be drawn: each run of the computer simulation increases the chances of achieving better solutions. The aim of the study is not to fine-tune the parameters of the genetic algorithm, therefore only one computer simulation is run for each data set for further research.

Table 3 presents the best multiple determination coefficients obtained for the regression coefficients $\left(b_{0}, b_{1}, \ldots, b_{6}\right)$ in the simulation described by the triple: $\mathrm{chn}$, in and $\mathrm{mn}$. The values of the multiple determination coefficients should be in the range $[0,1]$.

The phenomenon of high values of the multiple determination coefficients can be explained by the achieved values of the

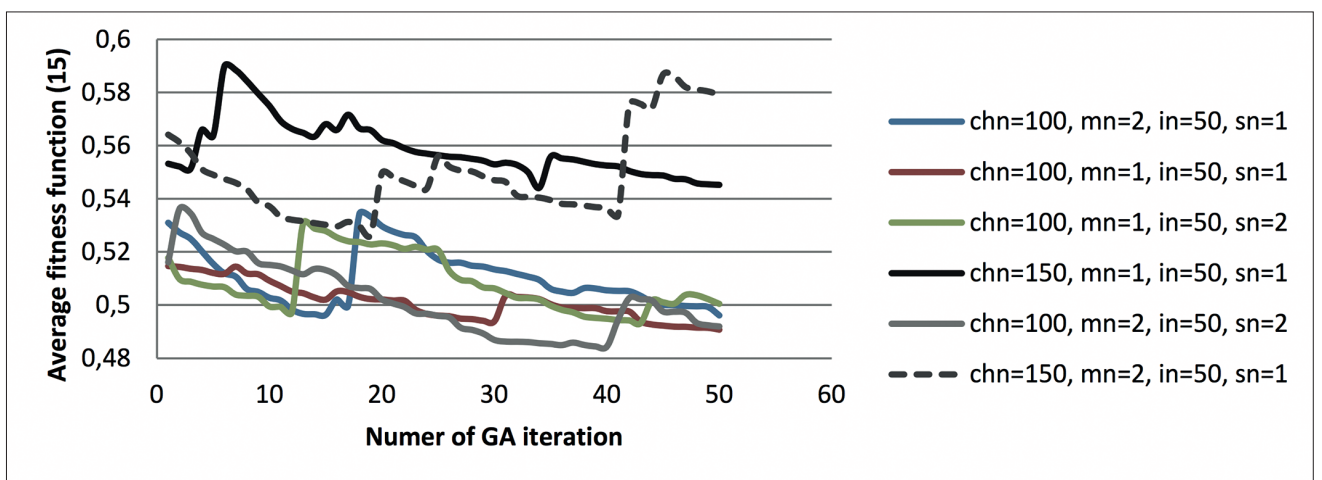

Fig. 3. The average fitness function obtained in iteration $1,2,3, \ldots, 50$ for the simulation described by: chn=\{100,150\}, in $=50, m n=\{1,2\}$ and sn $=\{1,2\}$

Tab. 3. The best multiple determination coefficient obtained for regression coefficients $\left(b_{0}, b_{1} \ldots, b_{6}\right)$ in the simulation described by: $c h n=\{100,150\}, i n=50, m n=\{1,2\}$ and $s n=\{1,2\}$

\begin{tabular}{|c|c|c|c|c|c|c|c|c|c|c|c|}
\hline chn & $\mathrm{mn}$ & in & sn & $R\left(\rho_{\eta}\right)^{2}$ & $b_{0}$ & $b_{1}$ & $b_{2}$ & $b_{3}$ & $b_{4}$ & $b_{5}$ & $b_{6}$ \\
\hline 100 & 2 & 50 & 1 & 97.6425 & -0.9251 & 0.000986 & 0.001069 & 0.001127 & 0.000274 & 0.0835 & 4.0258 \\
\hline 100 & 1 & 50 & 2 & 223.799 & 1.0003 & $8.16 \mathrm{e}-05$ & 0.000172 & 0.00255 & 0.000193 & $3.5 e-05$ & -7.999 \\
\hline 150 & 1 & 50 & 1 & 196.203 & -0.999127 & 0.000448 & 0.0024 & 0.000695 & 0.000966 & 0.01064 & 0.0902 \\
\hline 100 & 2 & 50 & 2 & 134.126 & -0.9103 & 0.000964 & 0.00258 & 0.00081 & 0.000559 & 0.00615 & 0.0874 \\
\hline 150 & 2 & 50 & 1 & 153.13 & -0.999984 & 0.00066 & 0.000551 & 0.000277 & $9.64 \mathrm{e}-05$ & 0.000595 & 9.00015 \\
\hline
\end{tabular}




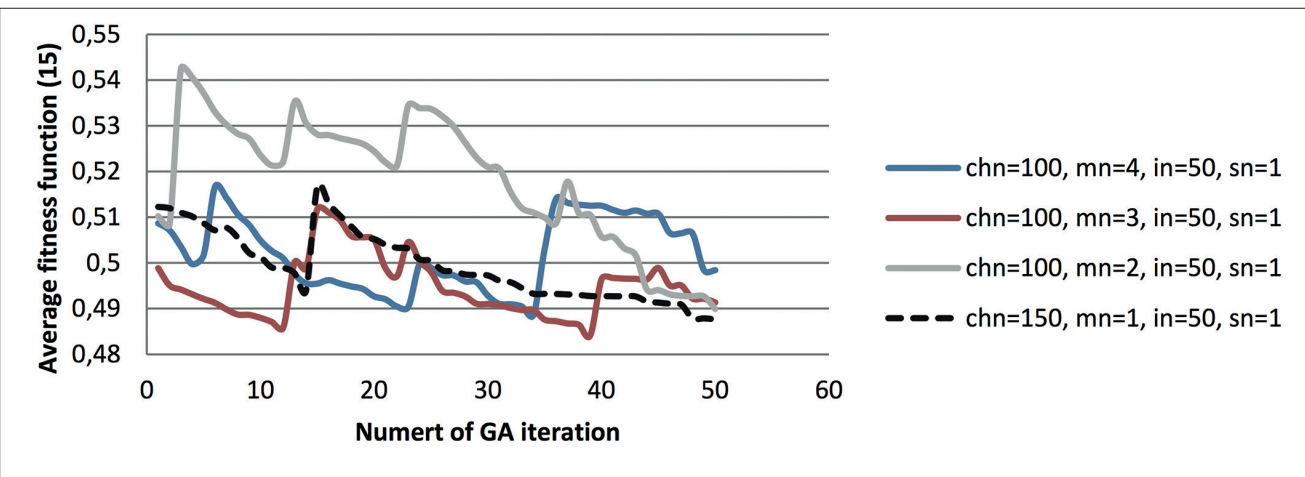

Fig. 4. The average fitness function obtained in iteration $1,2,3, \ldots, 50$ for the simulation described by the triple: chn, mn et al. after modifying the range for parameter $b_{0}$

Tab. 4. The best multiple determination coefficient obtained for regression coefficients $\left(b_{0}, b_{1} \ldots, b_{6}\right)$ in the simulation described by the triple: chn, mn et al. after modifying the range for parameter $b_{0}$

\begin{tabular}{|c|c|c|c|c|c|c|c|c|}
\hline $\mathrm{mn}$ & $R\left(\rho_{\eta}\right)^{2}$ & $b_{0}$ & $b_{1}$ & $b_{2}$ & $b_{3}$ & $b_{4}$ & $b_{5}$ & $b_{6}$ \\
\hline 1 & 0.4247 & 0.0673 & $1.02 \mathrm{e}-05$ & $9.67 \mathrm{e}-05$ & $3.81 \mathrm{e}-05$ & $3.73 \mathrm{e}-05$ & 0.00846 & 0.00054 \\
\hline 2 & 1.01924 & 0.0482 & $6.27 \mathrm{e}-05$ & 0.000189 & 0.000369 & $3.89 \mathrm{e}-05$ & 0.0068 & 0.000937 \\
\hline 3 & $\mathbf{0 . 8 8}$ & $\mathbf{0 . 0 7 5 6}$ & $\mathbf{1 . 3 5 e - 0 5}$ & $\mathbf{1 . 3 9 e - 0 5}$ & $\mathbf{0 . 0 0 0 2 0 7}$ & $\mathbf{0 . 0 0 0 2 7 1}$ & $\mathbf{0 . 0 1 0 7 9}$ & $\mathbf{0 . 0 0 8 1 6}$ \\
\hline 4 & 0.6113 & 0.0619 & 0.000276 & $7.64 \mathrm{e}-05$ & $2.52 \mathrm{e}-05$ & $8.2 \mathrm{e}-06$ & $4.74 \mathrm{e}-05$ & $4 \mathrm{e}-06$ \\
\hline
\end{tabular}

parameters $\left\{b_{0} . . b_{6}\right\}$, which are randomly selected and far from the solution achieved by the Solver Appendix. In particular, parameter $b_{0}$ should reach values close to 15 (11), while the values achieved by GA are in the range [-0.9103, 1.003].

Based on the observed data for the regressin parameters, the following constrains are made for parameter $b_{0}$ : the value should be positive and within the range $[14,20]$ for the initial population.

After applying the knowledge of b0 values, a small number of experiments are repeated. Again, computer simulation $(\mathrm{cs}=1)$ is run for the values of the key parameters: the initial population $\{100,150\}$, the number of iterations $\{50\}$ and the number of mutation points $\{1,2,3,4\}$. The results are presented in Fig. 4 .

Now, the values of the multiple determination coefficients are in the range $[0,1]$ (except the second result) (Table 4 ). This means that the coding and differentiating procedures have been designed correctly. Parameter b0 reaches values in the range [0.0482, 0.0756] (Table 4). Due to this phenomenon, parameter $b_{0}$ does not dominate the multiple linear regression equation. Unlike the Solver Appendix, the GA predictions are more reliable.

The coefficient of multiple determination is very good, $R^{2}=0.88$, thus the achieved equation fits the historical data. The average time of repair for a ship depends on the pipeline repairing $(\mathrm{Pr})[\mathrm{m}]$ to the greatest extent. The regression coefficients $b_{0}, b_{1}, \ldots, b_{\mathrm{k}}$ are presented in Table 4 . The achieved regression equation is:

$$
\begin{gathered}
108 \cdot 0.756+0.00035 \sum_{i=1}^{n} H r_{i}+0.000393 \sum_{i=1}^{n} P a_{i}+ \\
0.000207 \sum_{i=1}^{n} S r_{i}+0.000271 \sum_{i=1}^{n} P l_{i}+0.01079 \sum_{i=1}^{n} P r_{i}+ \\
0.00816 \sum_{i=1}^{n} A s_{i}=\sum_{i=1}^{n} R T_{i}
\end{gathered}
$$

\section{RESULTS AND DISCUSSION}

In order to increase the value of the presented method for managers, further simulations are run with an emphasis on obtaining an accurate forecast. In the steps of the algorithms related to the selection, the possibility of replacing the existing chromosome (solution) with a new one is taken into account, provided that the standard deviation of the obtained forecasts is smaller (9). Results presented in the previous section were achieved using the coefficient of multiple determination (10) for selection pressure.

Computer simulation is run for the values of the key parameters: the initial population $\{100,50\}$, the number of iterations $\{50\}$ and the number of mutation points $\{1,2,3,4\}$; the results are presented in Table 5. The best solution is achieved for the multiple linear regression equation:

$$
\begin{gathered}
12.0028+1.1 e-06 \cdot H r_{i}+2.8 e-05 \cdot P a_{i}+ \\
5.97 e-05 \cdot \mathrm{Sr}_{i}+1.15 e-05 \cdot P l_{i}+ \\
0,00581 \cdot \mathrm{Pr}_{i}+0,000305 \cdot A s_{i}=R T_{i}
\end{gathered}
$$

Managers of Constanta Shipyard may substitute new data on planned works: hull repair $(\mathrm{Hr})$, painting $(\mathrm{Pa})$, structure renovation $(S r)$, panel renovation $(P l)$, pipe repair $(P r)$; on resources: dock (docking duration) and quayside (quaytime) and the age of the ship (As) into the equation (18), in order to estimate the future duration of maintenance. 
Tab. 5. The best standard deviation obtained for regression coefficients $\left(b_{0}, b_{1} \ldots, b_{6}\right)$ in the simulation described by the triple: chn $=\{50,100)$, in $=50, m n=\{1,2,3,4\}$

\begin{tabular}{|c|c|c|c|c|c|c|c|c|c|c|c|}
\hline $\mathrm{chn}$ & $\mathrm{mn}$ & $R\left(\rho_{\eta}\right)^{2}$ & $s\left(\rho_{\eta}\right)$ & $F\left(\rho_{\eta}\right)$ & $b_{0}$ & $b_{1}$ & $b_{2}$ & $b_{3}$ & $b_{4}$ & $b_{5}$ & $b_{6}$ \\
\hline 100 & 1 & 0.411959 & 2.79 & 0.50 & 9.00005 & $3.17 \mathrm{e}-05$ & $6.64 \mathrm{e}-05$ & 0.000224 & $7.71 \mathrm{e}-05$ & 0.00856 & 0.01057 \\
\hline 50 & 1 & 2.96448 & 5.76 & 0.50089 & 2.99923 & $3.79 \mathrm{e}-05$ & 0.000426 & 0.000107 & $2.72 \mathrm{e}-05$ & 0.00611 & 0.00101 \\
\hline 50 & 2 & 0.135776 & 2.767 & 0.500496 & 13.0006 & $3.35 \mathrm{e}-05$ & $3.5 \mathrm{e}-06$ & $4.1 \mathrm{e}-05$ & $3.53 \mathrm{e}-05$ & $6.91 \mathrm{e}-05$ & 0.00347 \\
\hline $\mathbf{5 0}$ & $\mathbf{3}$ & $\mathbf{0 . 2 3 1 7 8 4}$ & $\mathbf{2 . 6 4 1 3 9}$ & $\mathbf{0 . 5 0 3 4 0 3}$ & $\mathbf{1 2 . 0 0 2 8}$ & $\mathbf{1 . 1 e - 0 6}$ & $\mathbf{2 . 8 e}-\mathbf{0 5}$ & $\mathbf{5 . 9 7 e - 0 5}$ & $\mathbf{1 . 1 5 e - 0 5}$ & $\mathbf{0 . 0 0 5 8 1}$ & $\mathbf{0 . 0 0 0 3 0 5}$ \\
\hline 50 & 4 & 1.61047 & 3.6682 & 0.501773 & 12.0006 & $1.64 \mathrm{e}-05$ & $9.18 \mathrm{e}-05$ & 0.000216 & $3.35 \mathrm{e}-05$ & 0.0029 & 0.000486 \\
\hline
\end{tabular}

The multiple determination coefficient $R^{2}$ equals 0.42 and the standard deviation s equals 2.78 , for the regression coefficient values achieved using EXCEL (11). The solution with similar qualities was achieved using the GA with the multiple determination coefficient $R^{2}$ equal to 0.41 and the standard deviation $s$ equal to 2.79 (first row, Table 5). An interesting solution was achieved using the GA, with a lower value of the standard deviation, $s=2.64$, and a lower value of the multiple determination coefficient, $R^{2}=0.23(18)$. The average time of repair for a ship depends on the pipeline repairing $(P r)$ and the age of the ship (As), to a large extent (18).

The maintenance times estimated for the regression coefficient values described in the fourth row in Table 5, using the GA and EXCEL for the regression coefficient values described in Eq. (11) and historical data on ships' repair times, are presented in Fig. 5.

By analysing the achieved results (Fig. 5) the following conclusion can be given:

- the adjustment of the regression line to the empirical data is small in both cases (it is of the order of 5\%). The GA achieved the slightly better adjustment of the regression line to the empirical data $\left(R^{2}=0.055\right)$ compared to $\operatorname{EXCEL~}\left(R^{2}=0.052\right)$. - it is clear that, in both graphs, the estimated repair time is very sensitive to the increase in 'historical' time: a relatively small increase in 'historical' time gives a more pronounced increase in the estimated time.

- as for the difference in the dispersion of points in the case, then, in the case of GA, all variability is in the range $(12,16)$; in the case of using analytical calculations in Excel, a small percentage 'protrudes' beyond this range. This is probably related to the fact that the analytical form of the forecast in regression gives an average estimation of an unknown value (regression estimation is the so-called mean estimate). Thus, the scattering of 'historical' values (some slight outlier values) can affect predictions using the regression function.

Further investigations are needed in order to increase the adequacy of the regression line to the empirical data.

\section{EFFECT OF THE NUMBER OF ITERATIONS ON THE FITNESS FUNCTION}

The following hypothesis is set: the number of iterations affects the quality of the obtained solutions (15).

First, two computer simulations $\{1,2\}$ are performed for the values of the key algorithm parameters: the initial population $\{100\}$, the number of iterations $\{100\}$ and the number of mutation points $\{3,4\}$. The mean population affinity function (obtained for each of the iterations) is presented in Fig. 6.

Observing the mean of the population affinity function obtained for the variable values of the mutation parameter $\{1,2$, 3,4 , the constant size of the initial population $=50$ (Fig. 4), the results obtained for the variable value of the mutation parameter $\{3,4\}$ and the size of the initial population $=100$ (Fig. 6), the number of mutation points $=3$ is selected for further computer simulations.

Further computer simulations are run for the values of the key parameters: size of the initial population $\{100\}$, the number of iterations $\{100,200,300,400,500,600,700\}$ and the

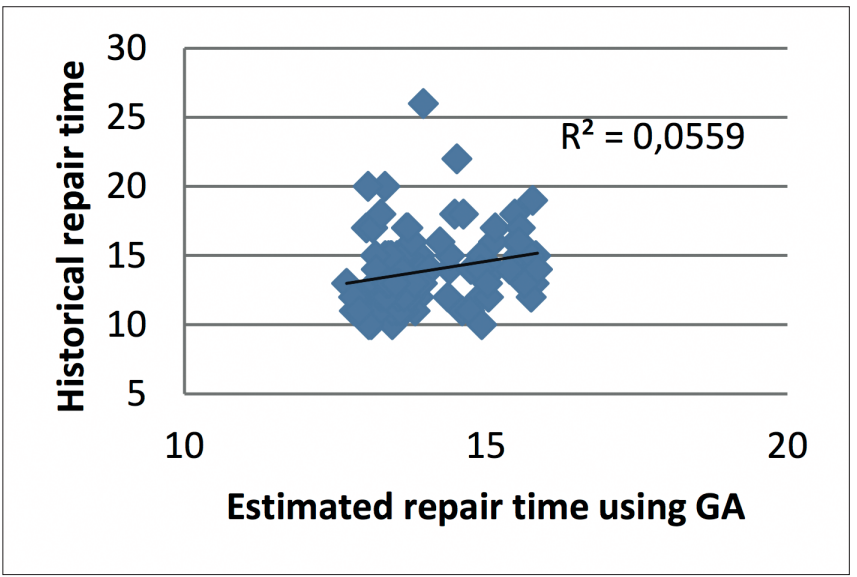

a)

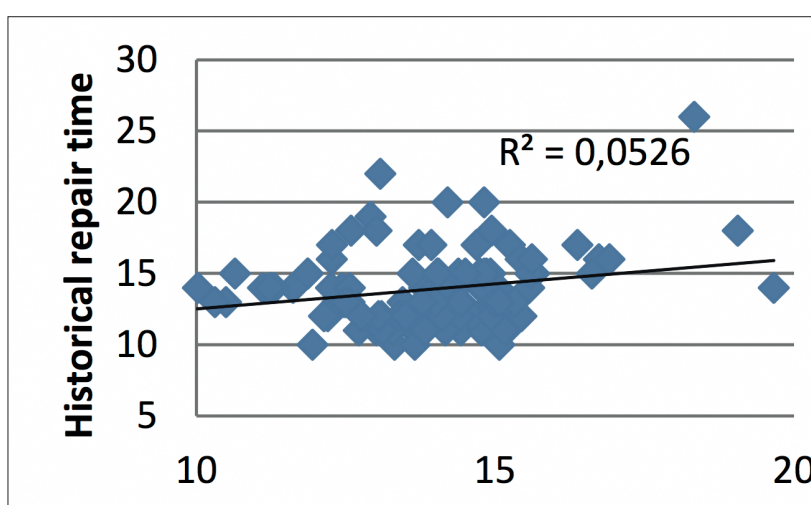

Estimated repair time using EXCEL

Fig. 5. Variation of estimated ship repair time (days) depending on historical repair time. Data dispersion and interpolation line for estimated repair time achieved using a) GA; b) EXCEL 


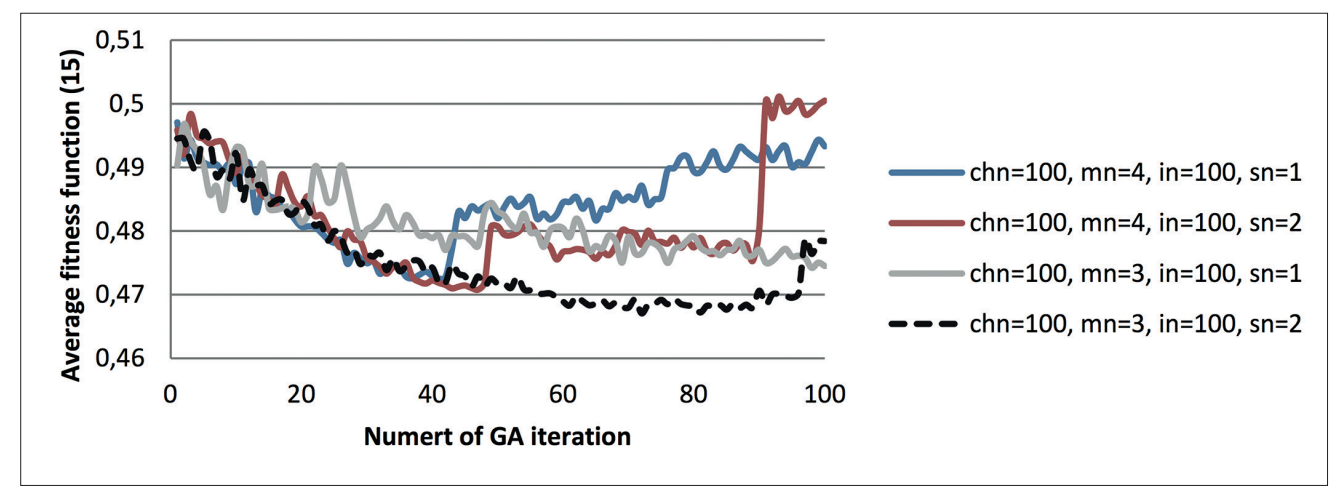

Fig. 6. The average fitness function obtained in iterations for the simulation described by: chn=100, in $=100, m n=\{3,4\}$ and $s n=\{1,2\}$

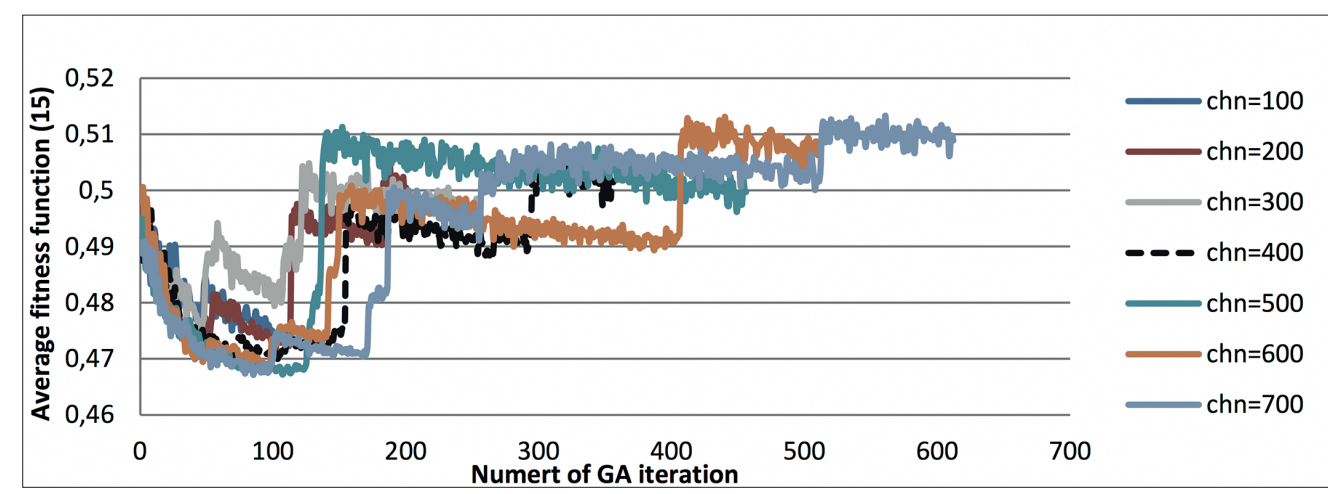

Fig .7. The average affinity function obtained by iteration for the simulation described by the triple: chn=100, in $=\{100,200,300,400,500,600,700\}$ and $m n=3$

number of mutation points $\{3\}$. The mean population affinity function (obtained for each of the iterations) for each computer simulation is presented in Fig. 7. By analysing the achieved results, the following conclusions can be given:

- the average affinity function of the population improves up to the number of iterations around 100 ;

- the average affinity function of the population depends on the maximal standard deviation and the maximal coefficient of multiple determination achieved in a population (15).

The effect of the number of iterations on the standard deviation value and the multiple determination coefficient value is presented in Fig. $8 \mathrm{a}$ and $8 \mathrm{~b}$, respectively. The standard deviation value improves with the number of iterations, from 100 to 500 (Fig. 8a). The multiple determination coefficient value improves with the number of iterations from 100 to 400 (Fig. 8b).
We can analyse the solution achieved for the key parameters: the initial population $\{100\}$, the number of iterations $\{500\}$ and the number of mutation points $\{3\}$ (Fig. 8). The solution (19) was achieved for the value of the standard deviation, $s=2.42$ and the value of the multiple determination coefficient, $R^{2}=0.19$.

$$
\begin{gathered}
12.00211+2.37 e-05 \cdot H r_{i}+1.05 e-05 \cdot P a_{i}+ \\
0.000269 \cdot S r_{i}+6.1 e-06 \cdot P l_{i}+0,00451 \cdot P r_{i}+ \\
8.9 e-0,5 \cdot A s_{i}=R T_{i}
\end{gathered}
$$

The average time of repair for a ship depends on the pipeline repairing $(P r)$ and the structure renovation $(S r)$ to a large extent (19).

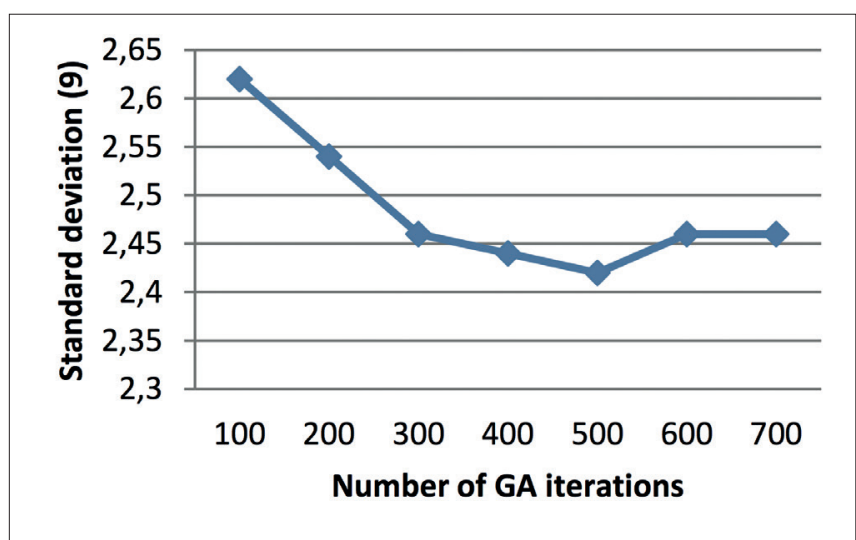

Fig. 8.

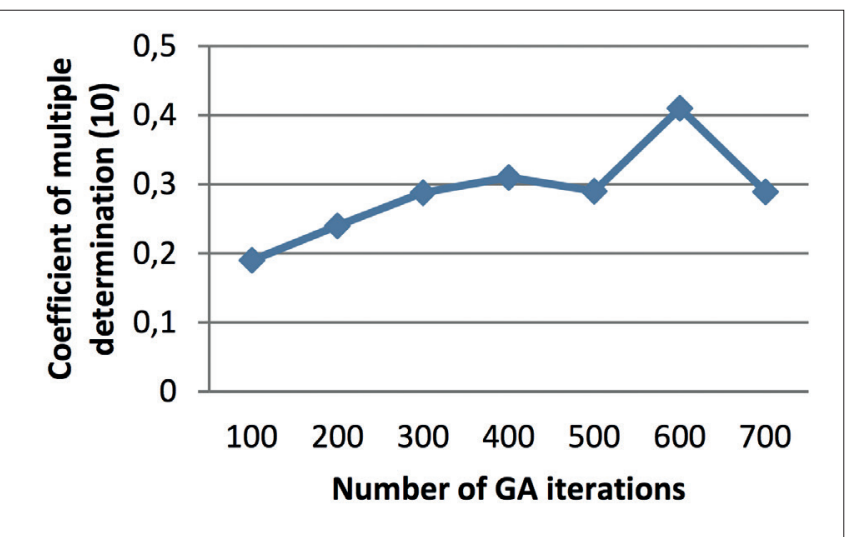

b) 
The variation of maintenance times estimated using the GA for the regression coefficient values described in equation (19) (depending on historical repair times) is presented in Fig. 9.

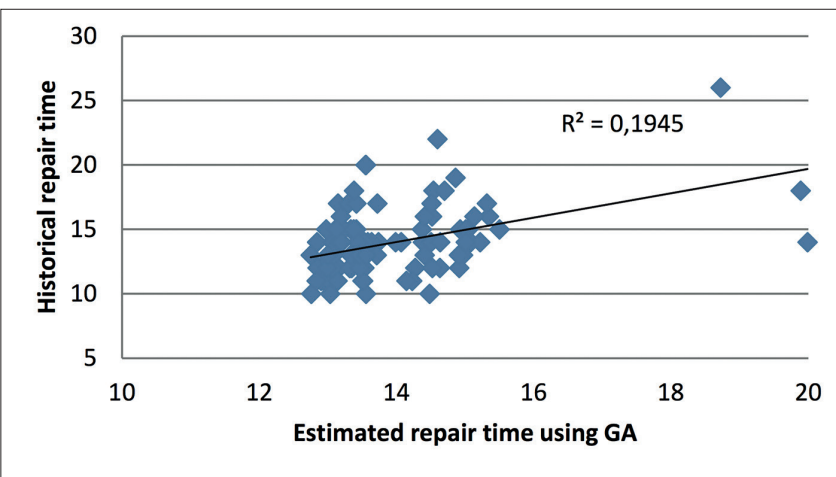

Fig. 9. Variation of estimated ship repair time (days) depending on historical repair time. Data dispersion and interpolation line for estimated repair time achieved using GA for the simulation described by the triple: $c h n=100$, in $=500$ and $m n=3$

By analysing the achieved results, the following conclusion can be given:

- the adjustment of the regression line to the empirical data is better (it is of the order of 19\%) compared to the solution achieved using EXCEL (it is of the order of 5\%) (11).

\section{CONCLUSIONS}

The paper presented the problem of reliable estimation of the duration of repair works on a ship during docking in a shipyard. Two computer programs were used for the problem: Microsoft Office Excel and the original genetic algorithm. The simulations performed, proved that the parameters achieved by the GA for multiple linear regression are more adequate. The parameters achieved by GA better described the relationship between dependent historical (repair time) and independent (ship type, age) data. The presented approach of the estimation of the maintenance duration is very promissing. The results significantly improved the adequacy of the presented model.

GA modifications will be the subject of future work to find an optimal solution. GAs are effective techniques for searching a solution space. However, chromosome diversity often decreases before the optimal solution is reached. This phenomenon is called premature algorithm convergence. Achieving better solutions in the previous population means that their clones are placed in the next population more frequently. They also tend to match with individuals with similar genes. These phenomena result in the loss of a diversity of solutions $[17,18]$. The way in which old individuals are replaced with new ones and a high selection pressure cause the algorithm to converge prematurely, therefore, many forms of hybridisation with local search algorithms have been proposed to improve the local search $[19,20]$. For the problem of estimating the mean time of ship repair, further research is needed on various forms of hybridisation of the genetic algorithm.

\section{REFERENCES}

1. P. Bzura. 'Diagnostic model of crankshaft seals'. Polish Maritime Research. 2019, Vol. 26, Issue: 3, 39-46.

2. A. Krystosik-Gromadzinska, W. Zenczak. 'Improvements to a fire safety management system'. Polish Maritime Research. 2019, Vol. 26, Issue 4, 117-123.

3. Ch. Gong, D. M. Frangpol, M. Cheng. 'Risk-based life-cycle optimal dry-docking inspection of corroding ship hull tankers. Engineering Structures. 2019, 195, 559-567.

4. J. Girtler. 'Limiting distribution of the three-state semimarkov model of technical state transitions of ship power plant machines and its applicability in operational decisionmaking. Polish Maritime Research. 2020, Vol. 27, Issue: 2, 136-144.

5. S. Wu, Y. Chen, Q. Wu, Z. Wang. 'Linking component importance to optimisation of preventive maintenance policy'. Reliability Engineering and System Safety. 2016, 146, 26-32.

6. D. Butler. 'A Guide to Ship Repair Estimates in Man-hours' 2012, DOI: 10710.1016/B978-0-08-098262-5.00008-18.

7. S. Muthia, Naffisah, I. Surjandari, A. Rachman, R.W.H. Palupi, 'Estimation of Dry Docking Maintenance Duration using Artificial Neural Network'. Int Journal of Computing, Communications \& Instrumentation Engg. 2014, Vol. 1, Issue 1, 2349-1477.

8. I. Surjandari, R. Novita. 'Estimation Model of Dry Docking Duration Using Data Mining. World Academy of Science, Engineering and Technology. 2013, Vol. 7.

9. E. Manea, M-G. Manea, 'The Influence of the Deadweight in the Projection of the Duration of the Maritime Ships Mentenancy Works', Advanced Engineering Forum 2019, 34, 292-299.

10. K. A. Dev, M. Saha. 'Modelling and Analysis of Ship Repairing Time'. Journal of Ship Production and Design. 2015, Vol. 31, No. 1, 1-8.

11. W. Tarełko.'Control model of maintainability level'. Reliability Engineering and System Safety. 1995, 47, 85-91.

12. Z. Bouayed, Ch.E. Penney, A. Sokri, T. Yazeck, 'Estimating Maintenance Costs for Royal Canadian Navy Ships', Scientific Report DRDC-RDDC-2017-R147.

13. J.E.C. Arroyo, V. A. Armentano. 'Genetic local search for multi-objective flowshop scheduling problems'. European journal of operational research. 2005, 167, 717-738. 
14. X. Cai, K. N. Li. 'A genetic algorithm for scheduling staff of mixed skills under multi-criteria. European Journal of Operational Research. 2000, 125, 359-369.

15. G. Cavory, R. Dupas, G. Goncalves. 'A genetic approach to solving the problem of cyclic job shop scheduling with linear constraints. European Journal of Operational Research. 2005, $161,73-85$.

16. I. Paprocka, C. Grabowik, W.M. Kempa, D. Krenczyk, K. Kalinowski. 'The influence of algorithms for basic-schedule generation on the performance of predictive and reactive schedules'. Conf. Series: Materials Science and Engineering. 2018, 400, 1757-8981, DOI:10.1088/1757-899X/400/2/022042.

17. S. Bertel, J.-C. Billaut. 'A genetic algorithm for an industrial multiprocessor flow shop scheduling problem with recirculation'. European Journal of Operational Research. 2004, 159, 651-662.

18. [18] M.E. Kurz, R.G. Askin. 'Scheduling flexible flow lines with sequence-dependent setup times'. European Journal of Operational Research. 2004, 159, 66-82.

19. R. Cheng, M. Gen, Y. Tsujimura. 'A tutorial survey of jobshop scheduling problems using genetic algorithms, part II: hybrid genetic search strategies', Computers \& Industrial Engineering. 1999, 36, 343-346.

20. J. F. Goncalves, J. J. de M. Mendes, M. G. C. Resende. 'A hybrid genetic algorithm for the job shop scheduling problem' European Journal of Operational Research. 2005, 167, 77-95.

\section{CONTACT WITH THE AUTHORS}

\section{Remus Zagan}

e-mail:zagan.remus@cmu-edu.eu

Constanta Maritime University

Faculty of Navigation and Naval Transport

104 Mircea cel Batran Street, 900663 Constanta

RoMANIA

\section{Iwona Paprocka}

e-mail: iwona.paprocka@polsl.pl

Silesian University of Technology

Faculty of Mechanical Engineering

Konarskiego 18A str., 44-100 Gliwice

Poland 